\title{
Interplay of shear and bulk viscosity in generating flow in heavy-ion collisions
}

\author{
Huichao Song* and Ulrich Heinz \\ Department of Physics, The Ohio State University, Columbus, Ohio 43210, USA
}

(Received 8 September 2009; published 10 February 2010)

\begin{abstract}
We perform viscous hydrodynamic calculations in $2+1$ dimensions to investigate the influence of bulk viscosity on the viscous suppression of elliptic flow in noncentral heavy-ion collisions at Relativistic Heavy Ion Collider energies. Bulk and shear viscous effects on the evolution of radial and elliptic flow are studied with different model assumptions for the transport coefficients. We find that the temperature dependence of the relaxation time for the bulk viscous pressure, especially its critical slowing-down near the quark-hadron phase transition at $T_{c}$, partially offsets effects from the strong growth of the bulk viscosity itself near $T_{c}$ and that even small values of the specific shear viscosity $\eta / s$ of the fireball matter can be extracted without large uncertainties from poorly controlled bulk viscous effects.
\end{abstract}

DOI: 10.1103/PhysRevC.81.024905

PACS number(s): $12.38 . \mathrm{Mh}, 25.75 . \mathrm{Ld}, 24.10 . \mathrm{Nz}$

\section{INTRODUCTION}

A question of widespread interest is that of the specific shear viscosity (shear viscosity per entropy density $\eta / s$ ) of the quark-gluon plasma (QGP) created in nuclear collisions at the Relativistic Heavy Ion Collider (RHIC). Ideal (i.e., inviscid) fluid dynamics has been quite successful in describing the transverse momentum $\left(p_{T}\right)$ spectra and the elliptic flow coefficient $v_{2}\left(p_{T}\right)$ of the bulk of the thousands of particles created in each, say, Au $+\mathrm{Au}$ collision [1]. The agreement between theory and experiments improves further when one interfaces a $(3+1)$-dimensional ideal-fluid description of the QGP phase with a hadron cascade during the late expansion stage, to properly account for the highly viscous evolution after hadronization of the QGP [3]. This success strongly suggests that the QGP fireball created at RHIC thermalizes very quickly and behaves like an almost-perfect liquid [4], which implies that it must be a strongly coupled plasma [5-7].

On the other hand, the quantum mechanical uncertainty relation places a fundamental lower bound on the specific shear viscosity of any medium [8], and explicit computation in a large class of very strongly coupled quantum field theories (unfortunately not including QCD) suggests that this limit is close to the so-called KSS bound $\left.\frac{\eta}{s}\right|_{\mathrm{KSS}}=\frac{1}{4 \pi} \approx 0.08[9,10]$. While this is a very small number (almost two orders of magnitude smaller than that of any other known (real) fluid $[10,11]$, with the possible exception of strongly interacting systems of ultracold fermionic atoms near the unitarity limit $[12,13])$, it is known that the anisotropic elliptic flow generated in noncentral relativistic heavy-ion collisions is very sensitive to shear viscosity $[14,15]$. The roots of this sensitivity lie in the exceedingly rapid expansion of the heavy-ion collision fireballs, especially during the early expansion stage, which is characterized by very large components of the velocity shear tensor [8]. Recent progress in performing causal relativistic hydrodynamical simulations of viscous fluids in $2+1$ dimensions [16-28] revealed that even very small specific shear viscosities, close to the KSS bound, should leave easily

*song@mps.ohio-state.edu identifiable experimental signatures, in particular, through a suppression of elliptic flow. In the experimental data this suppression is not large enough to lead to immediate failure of the ideal-fluid approach, suggesting that the QGP viscosity, in the temperature region probed by RHIC collisions, must also be close to the KSS bound [29-33].

Viscous hydrodynamics, in comparison with experimental data, allows in principle for an accurate determination of $\eta / s$. In practice, this requires excellent control of several other inputs that either are presently not known with sufficient accuracy or have not yet been correctly implemented in the numerical simulations [34]. The largest prevailing uncertainty is related to the initial source deformation that drives the elliptic flow, which is presently not known to better than $20 \%-30 \%[2,22,35,36]$ (see, however, recent suggestions for eliminating this error source [37,38]). As shown in Ref. [22], this leads at present to an $\mathcal{O}(100 \%)$ uncertainty in the extracted $\eta / s$ value. Two other effects of similar magnitude, which, however, work against each other and may largely cancel, are strong viscous effects [2] and the nonequilibrium chemical composition [39-43] in the late hadronic phase. Finally (and this is the point of the present paper), bulk viscous effects must be included in any study that aims to extract the specific shear viscosity $[34,44]$. However, even when making generous allowances for all these uncertainties, it appears clear that the effective shear viscosity-to-entropy ratio of the QGP, averaged over the expansion history of the fireballs created in RHIC collisions, cannot exceed the conservative upper limit,

$$
\left.\frac{\eta}{s}\right|_{\mathrm{QGP}}<5 \times \frac{1}{4 \pi} .
$$

This makes the QGP the most perfect liquid ever observed in the laboratory.

In the present paper we use the $(2+1)$-dimensional viscous relativistic fluid dynamic code VISH2+1 $[18,20,21]$ to study the effects of bulk viscosity and their interplay with shear viscosity in the buildup of radial and elliptic flow. Some preliminary results were reported in Refs. [34] and [44] (see also Ref. [45] for related work). Our work is preceded by three $(0+1)$-dimensional studies for systems undergoing boost-invariant longitudinal expansion without transverse flow 
[46-48] that explored the suggestion by Torrieri, Tomášik, and Mishustin [49] that, in rapidly expanding fireballs, bulk viscosity can lead to such large negative bulk pressures that the fluid becomes mechanically unstable against clustering and cavitation. Because bulk viscosity is expected to be maximal near the quark-hadron phase transition (see discussion in Sec. II), those studies predicted that bulk viscous effects become important mostly during the second half of the fireball expansion when the QGP undergoes hadronization. At that time the scalar expansion rate $\theta \equiv \partial_{\mu} u^{\mu}$ [where $u^{\mu}(x)$ is the flow four-velocity], which, for one-dimensional boostinvariant expansion, equals $\theta=1 / \tau$ (where $\tau=\sqrt{t^{2}-z^{2}}$ is the longitudinal proper time, with $z$ indicating the longitudinal or beam direction), is already small enough to significantly temper the growth (in magnitude) of the (negative) bulk pressure, leading to instability problems only for relatively large peak values for the specific bulk viscosity $\zeta / s$ [46-48].

Our work improves on these analyses by including a realistic initial transverse density profile and the resulting transverse flow in the fireball. This has two important consequences. (i) The transverse flow increases the expansion rate $\theta$, leading to larger bulk pressures $|\Pi|$ for a given $\zeta / s$. (ii) Some of the matter near the dilute transverse edge of the fireball experiences large bulk viscosities already at very early times where the expansion rate $\theta \sim 1 / \tau$ is high. This leads to much more severe problems with mechanical instability in VISH $2+1$ than for simple one-dimensional expansion and to correspondingly smaller values for the upper limit of $\zeta / s$ that allows for stable hydrodynamic evolution. Even more restrictive than the condition for mechanical stability is the self-consistency constraint for the validity of viscous hydrodynamics itself: the entire framework, which is based on a near-equilibrium expansion, breaks down when viscous corrections to the local equilibrium distribution function become comparable to the thermal equilibrium terms. We show that this happens, for particles with typical momenta $p \sim 3 T$, even before the effective total pressure becomes negative and mechanical instability sets in. ${ }^{1}$ While the formalism may be able to qualitatively indicate where and when cavitation sets in [46-48], we doubt that the phenomenon itself can be self-consistently described within the existing viscous hydrodynamic frameworks.

Obviously, viscous hydrodynamics can predict the viscous suppression of elliptic flow reliably only within its domain of validity. We therefore restrict our attention to the parameter range where the bulk viscous pressure stays everywhere sufficiently small that stable hydrodynamic evolution is ensured. Within that range (which we determine), we study the effects of bulk viscosity and of the microscopic relaxation time for

\footnotetext{
${ }^{1}$ This argument assumes that the fluid is sufficiently weakly coupled that a microscopic kinetic description in terms of quasiparticle distribution functions makes sense. We do not know how to generalize this argument to strongly coupled field theories that have no particle interpretation. We suggest that the criterion $\left(|\Pi|+\left|\pi^{\mu \nu}\right|\right) /(e+p) \ll 1$ (where $\left|\pi^{\mu \nu}\right|$ is the largest viscous pressure component) defines the domain of validity of (second-order) viscous hydrodynamics, irrespective of the microscopic structure of the fluid.
}

the bulk viscous pressure on radial and elliptic flow, with and without additional shear viscosity. For a fluid with constant specific shear viscosity $\eta / s=1 /(4 \pi)$, we find that, depending on initial conditions and details of the temperature dependence of the relaxation time, bulk viscosity increases the viscous suppression of $v_{2}\left(p_{T}\right)$ by $5 \%-50 \%$. This large range indicates not only that bulk viscosity is a potentially serious contaminant in the extraction of the specific shear viscosity $\eta / s$ from elliptic flow data, but also that a robust theoretical effort is needed to better constrain the range of reasonable values for the bulk viscosity and its associated relaxation time. We find that the uncertainty range is drastically reduced, to the 10\%-20\% level, if we impose proportionality between the specific bulk viscosity and its associated relaxation time, as indicated by kinetic theory. The critical growth of the specific bulk viscosity near the quark-hadron phase transition is then accompanied by critical slowing-down of the dynamics of the viscous bulk pressure. This diminishes the bulk viscous contribution to the viscous suppression of elliptic flow.

The paper is organized as follows. In Sec. II we review the present state of knowledge of the temperature dependence of the specific bulk and shear viscosities, $\zeta / s$ and $\eta / s$, and their associated microscopic relaxation times. Based on this analysis we introduce specific parametrizations for $(\zeta / s)(T)$ and the bulk pressure relaxation time $\tau_{\Pi}(T)$, which we use later in the numerical simulations. Section III gives a brief summary of specific features of the viscous hydrodynamic equations solved in this work, referring to earlier work for a more general description. In Sec. IV we discuss generic effects of bulk and shear viscosity on the hydrodynamic evolution of fireball eccentricity and flow and their implications for the final hadron spectra and elliptic flow. Section V discusses the sensitivity of bulk viscous effects to the initialization of the bulk viscous pressure and to its relaxation time. In Sec. VI we explore the range of bulk viscosities that allows for stable viscous hydrodynamic evolution. Consequences of bulk viscous effects for the extraction of the specific shear viscosity $\eta / s$ from experimental elliptic flow data are discussed in Sec. VII, before we summarize our findings in Sec. VIII.

\section{VISCOSITIES AND RELAXATION TIMES}

The present state of knowledge of the viscous properties of strongly interacting matter at high temperatures is nicely reviewed in Ref. [50], to which we refer for details. Kinetic theory [51] and experiment [10,11] show that, for nonrelativistic fluids, the specific shear viscosity $\eta / s$ typically reaches a minimum near the liquid-gas phase transition, rising both toward lower temperatures in the liquid phase and toward higher temperatures in the gas phase. Lattice QCD [52], perturbative QCD [53], and hadron cascade simulations [54] indicate that relativistic QCD matter behaves analogously, but with the liquid and gas phases interchanged (the liquid QGP phase exists at higher temperatures than the hadronic gas phase). According to perturbative [53] and lattice [52] QCD, the increase in $\eta / s$ with temperature in the QGP phase is weak over the temperature range explored in RHIC collisions, suggesting the use of a constant $\eta / s$ for the 
QGP in hydrodynamic simulations. Here we use the smallest value for this constant permitted by the KSS conjecture [10], $\left.\frac{\eta}{s}\right|_{\mathrm{KSS}}=\frac{1}{4 \pi} \approx 0.08$, to extract reasonable upper bounds for the uncertainties introduced by bulk viscous effects into the extraction of such a small value from experimental data. (We note that the assumption of a constant $\eta / s$ is unacceptable for quantitative attempts to extract it from heavy-ion collision data; at least, one must account for a significant increase in this ratio during hadronization and in the late hadronic phase [54].)

The relaxation time $\tau_{\pi}$ for the shear viscous pressure tensor $\pi^{\mu \nu}$ has been computed for a relativistic Boltzmann gas [55,56], in weakly coupled QCD [57], in lattice QCD [58], and in $\mathcal{N}=4 \mathrm{SYM}$ theory at infinite coupling [59-61]. The results can be presented in the form $\tau_{\pi}=\frac{\lambda}{T} \frac{\eta}{s}$, with $\lambda$ bracketed by $2 \lesssim \lambda \lesssim 6$. Here we use $\tau_{\pi}=\frac{3}{T} \frac{\eta}{s}=\frac{1.5}{2 \pi T}$.

Theoretical knowledge of the specific bulk viscosity $\zeta / s$ is more murky. For a noninteracting system of massless quanta it vanishes exactly, owing to conformal invariance. Interactions lead to deviations from zero that usually remain small, except near phase transitions, where the system may develop large correlation lengths [62-64]. Kinetic theory gives $\zeta / \eta=\kappa\left(\frac{1}{3}-\right.$ $\left.c_{s}^{2}\right)^{2}$, where $\kappa=5 / 3$ in relaxation time approximation [66] and $\kappa=15$ for a system of photons radiated by massive particles in thermal equilibrium [67]. The complete leading-order result for weakly coupled QCD [68] is roughly consistent with the latter of these two but adds a weak (decreasing) temperature dependence. At high temperatures $c_{s}^{2} \approx \frac{1}{3}$, so the ratio $\zeta / \eta$ is small, of second order, in the deviation. For strongly coupled $\mathcal{N}=4 \mathrm{SYM}$ theory one obtains a lower bound for this ratio that is only linear in this deviation and thus much larger: $\zeta / \eta \geqslant$ $2\left(\frac{1}{3}-c_{s}^{2}\right)$ [69]. For the hadron gas different authors [70-72] agree that $\zeta / \eta \ll 1$ just below $T_{c}$ and that this ratio decreases toward lower temperatures. There is no agreement on the sign of the temperature dependence of the specific bulk viscosity $\zeta / s$ itself, which, according to Ref. [70], increases toward lower temperature for massive pions but decreaes for massless pions [72]. However, there are general arguments $[63,73]$ that support the idea that $\zeta / s$ should peak near the quark-hadron phase transition, owing to long-range correlations related to the restoration of chiral symmetry; at a second-order critical point $\zeta / s$ is predicted to diverge $[64,65]$.

We assume here that $\zeta / s$ quickly approaches zero once $T$ decreases below $T_{c}$; above $T_{c}$, we parametrize it as $\frac{\zeta}{s}=\frac{1}{2 \pi}\left(\frac{1}{3}-\right.$ $c_{s}^{2}$ ) [which corresponds to the Buchel bound [69] for $\eta / s=$ $1 /(4 \pi)]$, using $c_{s}^{2}(T)$ extracted from the same lattice QCD data [74] that we used for our equation of state (EOS L; see Ref. [21] for details). The factor $\left(\frac{1}{3}-c_{s}^{2}\right)$ increases as we approach $T_{c}$ from above; the resulting increase in $\zeta / s$ is qualitatively, but not quantitatively, consistent with a direct extraction of $\zeta / s$ from lattice QCD [75] (for a critical discussion of this extraction see Ref. [64]) and with recent work in "holographic QCD" [76]. We connect our parametrization above $T_{c}$ to the assumed zero value for $\zeta / s$ well below $T_{c}$ by interpolating with a Gaussian function (see Fig. 1). This results in a peak value $(\zeta / s)\left(T_{c}\right) \simeq 0.04$ : about half as big as our choice for the (temperature-independent) specific shear viscosity $\eta / s$ and consistent with strong coupling estimates for strongly coupled conformal field theories using the AdS/CFT correspondence [77] (which was also used by Buchel in deriving his bound)

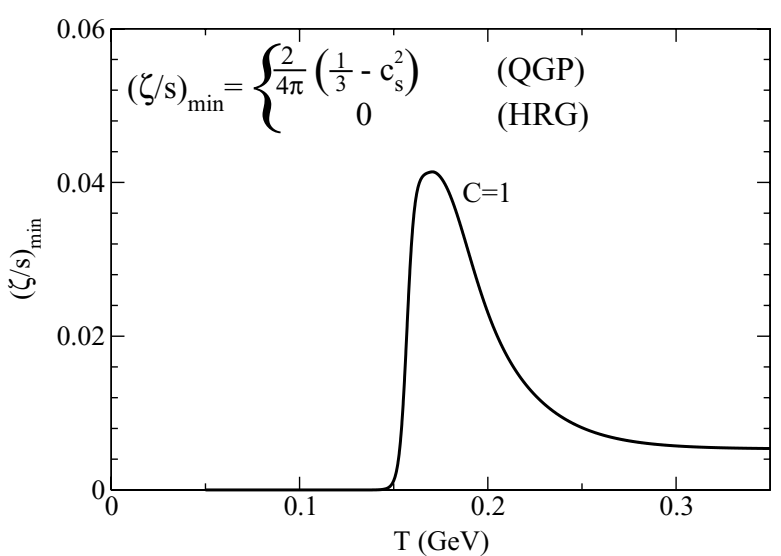

FIG. 1. Parametrization of the specific bulk viscosity $\zeta / s$ as a function of temperature. $C$ is a multiplicative scaling factor for the entire function; see text.

and with holographic QCD [76]. It is, however, more than 10 times smaller than both the lattice QCD value extracted by Meyer [75] and a recent AdS/CFT-based estimate by Buchel for a nonconformal plasma [65]. We will see that this factor 10 has crucial implications for the applicability of viscous hydrodynamics. To simulate larger bulk viscosities, we scale the function shown in Fig. 1 (which we refer to as "minimal bulk viscosity" for brevity) by a constant factor $C>1$.

Finally, we must specify the relaxation time $\tau_{\Pi}$ for the bulk viscous pressure $\Pi$, about which even less is known theoretically. In Israel-Stewart theory (in both its macroscopic and its microscopic kinetic formulation [55]), one has $\tau_{\Pi}=$ $\zeta \beta_{0}$, where $\beta_{0}$ is some combination of thermal equilibrium integrals. This suggests that, if $\zeta / s$ peaks near $T_{c}$, owing to growing correlation lengths, so does the relaxation time $\tau_{\Pi}$ for the bulk pressure ("critical slowing-down"). ${ }^{2}$ Buchel [65] found that in theories where the specific heat diverges at $T_{c}$, $c_{V} \sim 1 / \sqrt{\left|1-T_{c} / T\right|}$, the relaxation time can actually diverge at $T_{c}$ even if $\zeta / s$ remains finite, that is, as $T \rightarrow T_{c}, \tau_{\Pi}$ grows more strongly than $\zeta / s$ (see also the discussion in Ref. [64]). We use the parametrization

$$
\tau_{\Pi}(T)=\max \left[\tilde{\tau} \cdot \frac{\zeta}{S}(T), 0.1 \mathrm{fm}\right], \quad \text { with } \quad \tilde{\tau}=120 \mathrm{fm} / c .
$$

This increases linearly with $\zeta / s$ as $T \rightarrow T_{c}$ but imposes a nonzero lower bound on $\tau_{\Pi}$, for reasons of numerical stability of VISH $2+1$. For comparison we also study two constant relaxation time values, $\tau_{\Pi}=0.5$ and $5 \mathrm{fm} / c$, roughly corresponding to the smallest and largest values of Eq. (1) for temperatures $1 \leqslant T / T_{c} \leqslant 2$ if we set $C=1$.

\section{VISCOUS HYDRODYNAMICS}

We solve the following second-order viscous hydrodynamic equations ("Israel-Stewart equations" [55,78-80]),

$$
d_{\mu} T^{\mu \nu}=0, \quad T^{\mu \nu}=e u^{\mu} u^{\nu}-(p+\Pi) \Delta^{\mu \nu}+\pi^{\mu \nu},
$$

\footnotetext{
${ }^{2}$ We thank K. Dusling for a personal communication on this point.
} 


$$
\begin{aligned}
\Delta^{\mu \alpha} \Delta^{\nu \beta} D \pi_{\alpha \beta}= & -\frac{1}{\tau_{\pi}}\left(\pi^{\mu \nu}-2 \eta \sigma^{\mu \nu}\right) \\
& -\frac{1}{2} \pi^{\mu \nu} \frac{\eta T}{\tau_{\pi}} d_{\lambda}\left(\frac{\tau_{\pi}}{\eta T} u^{\lambda}\right) \\
D \Pi= & -\frac{1}{\tau_{\Pi}}(\Pi+\zeta \theta)-\frac{1}{2} \Pi \frac{\zeta T}{\tau_{\Pi}} d_{\lambda}\left(\frac{\tau_{\Pi}}{\zeta T} u^{\lambda}\right)
\end{aligned}
$$

in the two transverse spatial directions and time $[(2+1)-d]$, implementing boost-invariant longitudinal expansion along the beam direction. We assume zero net baryon density and thus vanishing heat conductivity. Here $T^{\mu \nu}$ is the energy momentum tensor, $\pi^{\mu \nu}$ is the shear pressure tensor, and $\Pi$ is the bulk pressure. $d_{\mu}$ denotes the covariant derivative components (see Refs. [16] and [24] for details) in the curvilear coordinates $\left(\tau, x, y, \eta_{s}\right.$ ), where $\tau=\sqrt{t^{2}-z^{2}}$ is the longitudinal proper time and $\eta_{s}=\frac{1}{2} \ln [(t+z) /(t-z)]$ is the space-time rapidity. $\Delta^{\mu \nu}=g^{\mu \nu}-u^{\mu} u^{\nu}$ projects onto the spatial components in the local rest frame [here $g^{\mu \nu}=\operatorname{diag}\left(1,-1,-1,-1 / \tau^{2}\right)$ is the metric tensor in $\left(\tau, x, y, \eta_{s}\right)$ coordinates]; $\nabla^{\mu}=\Delta^{\mu v} d_{v}$ is the spatial gradient, and $D=u^{\mu} d_{\mu}$ is the time derivative in that frame. The driving forces for the shear and bulk viscous pressures are the (symmetric and traceless) velocity stress tensor $\sigma^{\mu \nu}=\nabla^{\langle\mu} u^{\nu\rangle} \equiv \frac{1}{2}\left(\nabla^{\mu} u^{\nu}+\nabla^{\nu} u^{\mu}\right)-\frac{1}{3} \Delta^{\mu \nu} \theta$ and the scalar expansion rate $\theta=d_{\nu} u^{\nu}=\nabla_{\nu} u^{\nu}$, respectively. The shear and bulk viscosities $\eta$ and $\zeta$ and their associated relaxation times $\tau_{\pi}$ and $\tau_{\Pi}$ have been discussed in the preceding section.

The last terms in Eqs. (3) and (4) are of second order in deviations from local equilibrium. For conformal systems they can be written in various equivalent forms, up to higher-order corrections [21]. Even for nonconformal systems, such as QCD with the EOS L used here (see later), the difference between the terms as written here and their various conformal approximations are numerically insignificant [21] unless inconsistently large relaxation times are used. Other second-order terms that should be allowed for on the right-hand sides of Eqs. (3) and (4) were identified in Refs. [59] and [81], and some of their coefficients were derived in the weak coupling limit in Ref. [57]. Recent code verification efforts by the TECHQM Collaboration [82] indicate that these additional terms have very little numerical influence. We therefore ignore them in the present study.

The explicit form of Eqs. (2)-(4) for longitudinally boost-invariant (i.e., $\eta_{s}$-independent) systems is given in Ref. [16]. The equations are closed by providing an equation of state, for which we use EOS L as described in Ref. [21]. We study $\mathrm{Au}+\mathrm{Au}$ collisions with the same initial conditions for the starting time, $\tau_{0}=0.6 \mathrm{fm} / c$, and Glauber model initial energy density profiles as in Ref. [21], with peak density $e_{0} \equiv e\left(r=0, \tau_{0} ; b=0\right)=30 \mathrm{GeV} / \mathrm{fm}^{3}$ in central $(b=0)$ collisions. For the viscous pressures we use either zero $\left[\Pi\left(x, y, \tau_{0} ; b\right)=\pi^{\mu \nu}\left(x, y, \tau_{0} ; b\right)=0\right]$ or Navier-Stokes initial conditions $\left[\Pi\left(x, y, \tau_{0} ; b\right)=-\zeta \theta\left(\tau_{0}\right)\right.$ and $\left.\pi^{\mu \nu}\left(x, y, \tau_{0} ; b\right)=2 \eta \sigma^{\mu \nu}\left(\tau_{0}\right)\right]$, calculated from the initial velocity profile (which does not depend on $x, y$, and $b$, owing to the absence of initial transverse flow). The actual choice is noted in the discussion of the results. As in Ref. [21] we end the hydrodynamic evolution and compute the final hadron spectra on a freeze-out surface of constant temperature $T_{\text {dec }}=130 \mathrm{MeV}$.

\section{VISCOUS EVOLUTION AND SPECTRA: GENERIC FEATURES}

In this section we compare generic effects on the hydrodynamic evolution and final particle spectra caused by shear and bulk viscous effects separately. (Their combined effects are explored in Sec. V to Sec. VII.) To this end we perform hydrodynamic comparison runs for central $(b=0)$ and noncentral $(b=7 \mathrm{fm}) \mathrm{Au}+\mathrm{Au}$ collisions, using identical initial and freeze-out conditions, for (i) an ideal fluid, (ii) a viscous fluid with only minimal shear viscosity, $\frac{\eta}{s}=\frac{1}{4 \pi}$, and (iii) a viscous fluid with only "minimal bulk viscosity" $(C=1)$ as defined in Sec. II. In the viscous runs we choose Navier-Stokes initial conditions for the viscous pressures and equal relaxation times, $\tau_{\pi}=\tau_{\Pi}=3 \eta / s T$. (As a caveat we note that in the bulk viscous case, results can depend sensitively on the initial conditions, depending on the characteristics of the relaxation time for the bulk viscous pressure; see discussion in Sec. V.) The results for case (ii) supplement those for the smaller $\mathrm{Cu}+\mathrm{Cu}$ collision system studied in Refs. [18] and [20] [although for a more realistic equation of state and using Eq. (3) instead of the "simplified Israel-Stewart equation" employed in those earlier papers].

\section{A. Hydrodynamic evolution}

Figure 2(a) shows the time evolution of the local temperature in central $\mathrm{Au}+\mathrm{Au}$ collisions for the three cases. [We plot the temperature at a radius $r=3 \mathrm{fm}$ from the fireball center, as at $r=0$ the curves for cases (i) and (iii) are almost indistinguishable.] Compared with the ideal fluid, shear viscosity reduces the work done by longitudinal pressure and thus slows down the cooling process during the early stage; during the middle and late stages, shear viscosity accelerates the cooling, as the positive transverse shear pressure leads to stronger radial flow than for the ideal fluid [see Fig. 2(b) and Ref. [20] for a full discussion]. At late times, the shear viscous fireball thus cools more rapidly than the ideal fluid $[18,20]$.

Bulk viscosity, on the other hand, reduces the work done in all three directions, owing to the isotropic negative bulk pressure $\Pi \sim-\zeta \theta$ driven by the positive expansion rate $\theta>0$. As a result, radial flow develops less rapidly than for the ideal fluid [Fig. 2(b)], and the bulk viscous fluid cools (slightly) more slowly than the ideal one during all expansion stages [Fig. 2(a)]. While the expansion rate is highest at very early times, the bulk viscosity then is very small throughout the fireball, except for a thin region near the transverse edge of the fireball where the matter is close to $T_{c}$; bulk viscous effects are therefore almost negligible until most of the matter cools down to $T_{c}$. At this time the longitudinal expansion rate has significantly decreased, but transverse expansion picks up some of the the slack, and we see significant bulk viscous effects on radial flow evolution between 5 and $10 \mathrm{fm} / c$. Surprisingly, the consequences for the cooling rate 

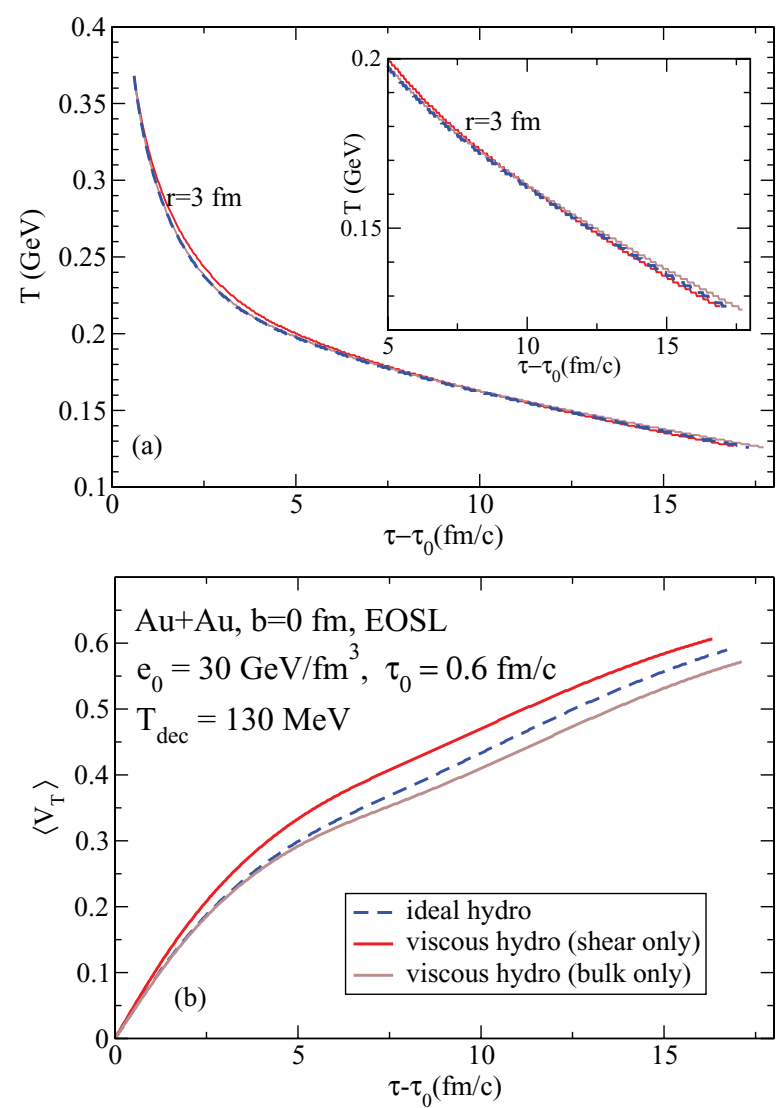

FIG. 2. (Color online) Time evolution of the local temperature (a) and average radial flow (b), from ideal hydrodynamics (dashed blue line), viscous hydrodynamics with only minimal shear (solid red line) or bulk (solid light-brown line) viscosity. In (b) the average radial flow is calculated with the Lorentz contracted energy density $\gamma_{\perp} e$ in the transverse plane as a weight function. The inset in (a) shows the late evolution with increased resolution.

are significantly smaller than in the shear viscous case: with the parameters studied here, the cooling rates for the ideal and bulk viscous fluids almost agree.

We now turn to noncentral collisions. To describe the fireball deformations in configuration and momentum space, we use the spatial eccentricity $\varepsilon_{x}=\frac{\left\langle y^{2}-x^{2}\right\rangle}{\left\langle y^{2}+x^{2}\right\rangle}$ (where $\langle\langle\cdots\rangle\rangle$ denotes an energy density weighted average over the transverse plane [83]) and the momentum anisotropies $\varepsilon_{p}=\frac{\left\langle T_{0}^{x x}-T_{0}^{y y}\right\rangle}{\left\langle T_{0}^{x x}+T_{0}^{y y}\right\rangle}$ (defined in terms of unweighted averages over the transverse plane of components of the ideal-fluid part of the energymomentum tensor and, thus, measuring only the collective flow anisotropy [20]) and $\varepsilon_{p}^{\prime}=\frac{\left\langle T^{x x}-T^{y y}\right\rangle}{\left\langle T^{x x}+T^{y y}\right\rangle}$ (defined in terms of the total energy-momentum tensor, which contains the viscous pressure components and thus additionally includes microscopic momentum anisotropies in the local rest frame of the fluid [20]).

Figure 3(a) shows the time evolution of the spatial eccentricity $\varepsilon_{x}$ for noncentral $\mathrm{Au}+\mathrm{Au}$ collisions at $b=7 \mathrm{fm}$. Compared with the ideal fluid, bulk viscosity decelerates, whereas shear viscosity initially accelerates, the decrease in the spatial eccentricity $\varepsilon_{x}$ with time. This is a direct consequence

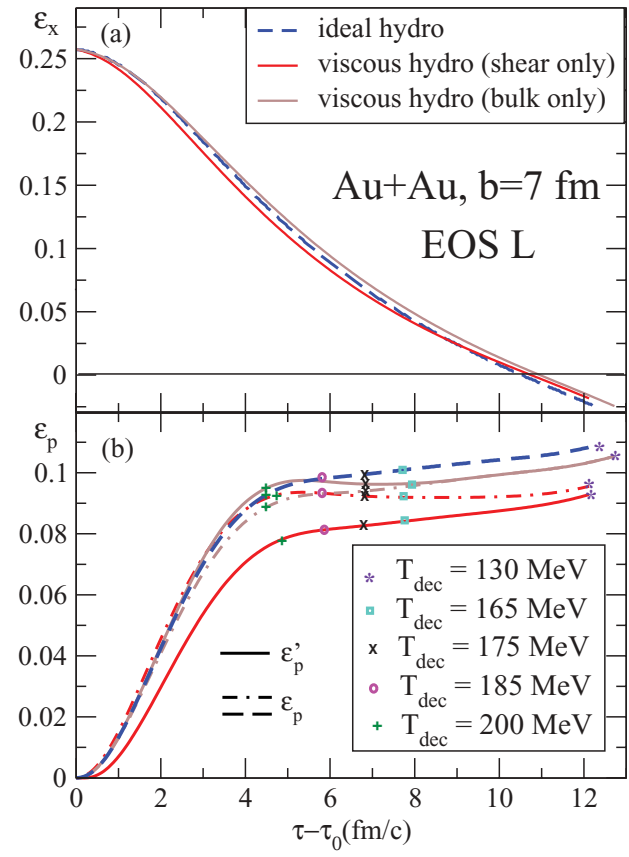

FIG. 3. (Color online) Time evolution of spatial eccentricity $\varepsilon_{x}$ (a) and momentum anisotropy $\varepsilon_{p}, \varepsilon_{p}^{\prime}$ (b), from ideal and viscous hydrodynamics (see text for details). In (b), the different symbols along the bulk viscous fluid lines indicate central $(r=0)$ freezeout times for different freeze-out temperatures as described in the key.

of the weaker radial flow in the bulk viscous case and the stronger radial flow in the shear viscous case. It is easy to see that isotropic radial expansion is enough to decrease the spatial eccentricity $\varepsilon_{x}$ [83]; anisotropic flow, with higher flow velocities in the reaction plane than perpendicular to it, only accelerates the rate at which it decreases. At late times, the eccentricity for the shear viscous fluid decreases more slowly than for the ideal one, as the ideal fluid develops stronger elliptic flow [see Fig. 3(b) and following discussion]. In contrast, in the bulk viscous case the slower rate of decrease in the eccentricity is caused by weaker radial flow.

As the spatial eccentricity of the fireball decreases, its momentum anisotropy increases. This is shown in Fig. 3(b). The dash-dotted lines for $\varepsilon_{p}$, which takes only the ideal-fluid part, $T_{0}^{\mu \nu}=(e+p) u^{\mu} u^{\nu}-p g^{\mu \nu}$, of the energy-momentum tensor into account, show how hydrodynamic forces convert the spatial anisotropy into a flow anisotropy. We observe that at early times the flow anisotropy $\varepsilon_{p}$ rides on the developing radial flow: compared to the ideal fluid, $\varepsilon_{p}$ develops a little faster in the shear viscous fluid but a little more slowly in the bulk viscous case, following the evolution of radial flow.

The difference between $\varepsilon_{p}$ (dash-dotted lines) and $\varepsilon_{p}^{\prime}$ (solid lines) stems from the viscous pressure components in the energy-momentum tensor. It reflects a contribution to the momentum anisotropy that arises not from anisotropic collective flow but from viscous deviations from isotropy of the microscopic momentum distribution, $f=f_{\text {eq }}+\delta f$, in the local fluid rest frame [20], accounted for by the nonideal terms 

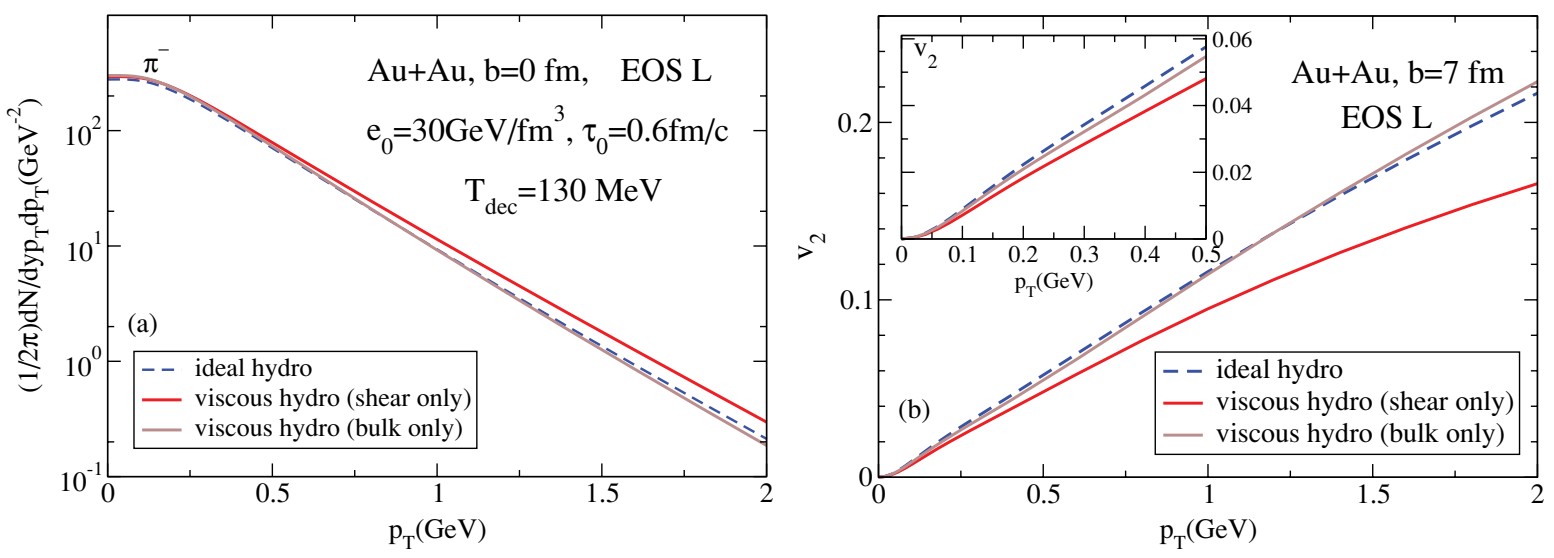

FIG. 4. (Color online) $p_{T}$ spectra and elliptic flow $v_{2}\left(p_{T}\right)$ for directly emitted pions (i.e., without resonance decay contributions).

in $T^{\mu \nu}$. Figure 3(b) shows that for the shear viscous fluid these viscous corrections are large and negative (reflecting local momentum anisotropies pointing out of the reaction plane [20], that is, opposite to the flow anisotropy), especially at early times, when the expansion rate and shear velocity components $\sigma^{\mu \nu}$ are large. In contrast, the bulk viscous fluid shows significant viscous corrections only after about $2.5 \mathrm{fm} / c$, lasting until about $8 \mathrm{fm} / c$, which is when, in these noncentral $(b=7 \mathrm{fm}) \mathrm{Au}+\mathrm{Au}$ collisions, the bulk of the matter passes through the phase transition where $\zeta / s$ is large [cf. the temperature markers on the curves shown in Fig. 3(b)]. The bulk viscous pressure contribution to $\varepsilon_{p}^{\prime}$ is positive, that is, pointing into the reaction plane, parallel to the collective flow anisotropy. (This was also recently pointed out by Monnai and Hirano [84].)

For both shear and bulk viscosity, the sign of the viscous pressure contributions to $\varepsilon_{p}^{\prime}$ obeys a "Lenz rule": they act against the radial-flow-driven effects on the momentum anisotropy. At late times these contributions become small in both cases: in the bulk viscous case, driven by the rapid disappearance of $\zeta$ below $T_{c}$ (as modeled by us), and in the shear viscous case, by the more gradual vanishing of the shear velocity tensor $\sigma^{\mu \nu}$ [20]. We see that in the bulk viscous case the radial flow effect on $\varepsilon_{p}^{\prime}$ eventually wins over that from the local deviation from equilibrium $\delta f$, whereas the opposite is true for the shear viscous fluid. In both cases, the net effect at freeze-out is thus a viscous suppression of the momentum anisotropy below the ideal fluid limit [see purple asterisks in Fig. 3(b)]. The viscous suppression of $\varepsilon_{p}^{\prime}$ resulting from shear viscosity is four to five times stronger than that arising from bulk viscosity.

\section{B. Spectra and elliptic flow}

From the hydrodynamic output at decoupling temperature $T_{\mathrm{dec}}$ the spectra and their azimuthal anisotropy, in particular, the elliptic flow coefficient $v_{2}\left(p_{T}\right)$, are computed with a modified Cooper-Frye algorithm that takes into account that in viscous hydrodynamics the local phase-space distribution $f(x, p)$ on the freeze-out surface deviates slightly from thermal equilibrium, $f=f_{\text {eq }}+\delta f$, owing to small but nonzero viscous pressure components $[15,20,85] .{ }^{3}$ Figure 4 (a) shows the pion $p_{T}$ spectra for central $\mathrm{Au}+\mathrm{Au}$ collisions from ideal and viscous hydrodynamics. Compared to the spectrum from ideal-fluid dynamics, shear viscosity leads to flatter spectra while bulk viscosity generates steeper ones. This is a direct reflection of the stronger radial flow caused by the positive transverse shear pressure and the weaker radial flow resulting from the negative bulk viscous pressure. The slightly larger normalization of the viscous spectra is a consequence of viscous entropy production, which leads to larger final multiplicities [21,85].

Figure 4(a) shows that shear and bulk viscosity act against each other in how they affect the slope of the $p_{T}$ spectra. When both viscosities are included together in the viscous calculations, this reduces the amount of readjustment needed in the initial conditions when fitting the measured transverse momentum spectra with viscous instead of ideal-fluid dynamics [85]. The differential elliptic flow $v_{2}\left(p_{T}\right)$ for soft pions, on the contrary, is affected by both bulk and shear viscosity in the same way: Fig. 4(b) shows that the viscous reduction of $\varepsilon_{p}^{\prime}$ in Fig. 3(b) translates directly into reduced elliptic flow $v_{2}$ in the final hadron spectra. This is true, in particular, in the low- $p_{T}$ region, $p_{T}<1 \mathrm{GeV} / c$ [see inset in Fig. 4(b)]. At larger $p_{T}$, the shear viscous $v_{2}$ suppression increases further, owing to a negative contribution from $\delta f$ along the freeze-out surface. In contrast, the bulk viscosity increases $v_{2}\left(p_{T}\right)$ above $1 \mathrm{GeV} / c$ because it results in steeper $p_{T}$ spectra. At $p_{T}=0.5 \mathrm{GeV} / c$ (approximately the mean transverse momentum for pions), we find that minimal bulk viscosity suppresses $v_{2}$ by $\sim 5 \%$, while minimal shear viscosity leads to a suppression of $\sim 20 \%$.

If this were the complete story, the additional $\sim 5 \%$ bulk viscous $v_{2}$ suppression would lead to an $\sim 25 \%$ reduction of the value for $\eta / s$ that one might extract from experimental elliptic flow data, by comparing them with an ideal-fluid dynamical baseline as proposed in Ref. [22]. This is a non-negligible

\footnotetext{
${ }^{3}$ We note that in our calculations the bulk viscous pressure $\Pi \approx 0$ on the $T_{\mathrm{dec}}=130 \mathrm{MeV}$ freeze-out surface, as our parametrization of the bulk viscosity gives $(\zeta / s)\left(T_{\mathrm{dec}}\right)=0$ (see Fig. 1). Accordingly, the bulk viscous pressure $\Pi$ does not contribute to the deviation $\delta f$ from local equilibrium on the freeze-out surface.
} 

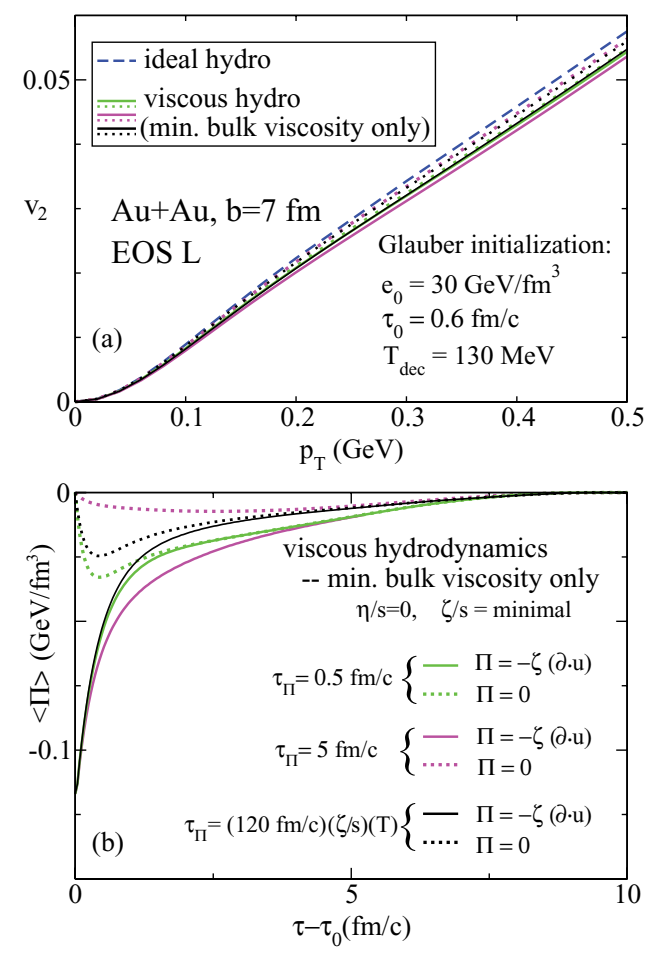

FIG. 5. (Color online) (a) Differential elliptic flow $v_{2}\left(p_{T}\right)$ for directly emitted pions (without resonance decays) from ideal and viscous hydrodynamics, including only minimal $(C=1)$ bulk viscosity. (b) Time evolution of the bulk pressure $\langle\Pi\rangle$ averaged over the transverse plane (weighted by the energy density) from viscous hydrodynamics. Different curves correspond to different initializations and relaxation times, as indicated (see text for discussion).

effect. Because the input for the bulk viscosity used in our calculations is fraught with large theoretical uncertainties, as discussed in Sec. II, this points to a likelihood for correspondingly large uncertainties in the empirical extraction of $\eta / s$ from the experiment. In Sec. $\mathrm{V}$ we follow this line of thought further, by investigating the additional sensitivity of bulk viscous effects to the initial conditions for the bulk viscous pressure and to its relaxation time.

\section{SENSITIVITY OF BULK VISCOUS DYNAMICS TO INITIAL CONDITIONS AND RELAXATION TIMES}

In this section we focus entirely on bulk viscosity and investigate what happens when we change the initial value for the bulk viscous pressure $\Pi$ and its relaxation time $\tau_{\Pi}$. We study only "minimal bulk viscosity" as defined in Sec. II (i.e., $C=1$ ), leaving the discussion of larger values to Sec. VI.

Figure 5 shows, for peripheral $\mathrm{Au}+\mathrm{Au}$ collisions at $b=7$ fm, the differential elliptic flow for pions [Fig. 5(a)] and the time evolution of the average value of the bulk viscous pressure $\langle\Pi\rangle$ [Fig. 5(b)], for the two initial conditions (zero and Navier-Stokes) for $\Pi$ and the three choices of relaxation time scales $\tau_{\Pi}$ discussed in Sec. II. At $p_{T}=0.5 \mathrm{GeV} / c$, Fig. 5(a) indicates a bulk viscous $v_{2}$ suppression that ranges (for $C=1$ ) from $\sim 2 \%$ to $\sim 10 \%$. For the short relaxation time
$\tau_{\Pi}=0.5 \mathrm{fm} / c$ (solid and dotted green lines), the suppression is insensitive to the initialization of $\Pi$, yielding about $8 \%$ suppression below the ideal-fluid value at $p_{T}=0.5 \mathrm{GeV} / c$ for both zero and Navier-Stokes initial values. Figure 5(b) explains the underlying reason for this observation: for this short relaxation time, $\langle\Pi\rangle$ quickly loses all memory of its initial value, relaxing for both initial conditions to the same trajectory after $\sim 1-2 \mathrm{fm} / c$ (i.e., after a few relaxation times, similar to what we saw earlier [20] for the shear pressure components). This also demonstrates that most of the finally observed bulk viscous $v_{2}$ suppression is generated during the middle part of the expansion, when most of the matter cools through the phase transition. If it were dominated by large negative bulk viscous pressures in the outer shell of the fireball at early times, we should see stronger sensitivity to the initial value for $\Pi$.

This changes completely if we choose a 10 times longer relaxation time, $\tau_{\Pi}=5 \mathrm{fm} / c$ (solid and dotted magenta lines in Fig. 5). Now the bulk viscous $v_{2}$ suppression becomes extremely sensitive to the initialization of $\Pi$. For zero initialization, the average bulk pressure $\langle\Pi\rangle$ always remains small, leading to very small $[\mathcal{O}(2 \%)]$ final suppression effects for $v_{2}$. For Navier-Stokes intialization, $\langle\Pi\rangle$ is initially very large and negative (owing to the large initial expansion rate), and instead of relaxing to smaller values as predicted by Navier-Stokes theory (and realized by the solid green line, corresponding to short $\tau_{\Pi}$ ), it remains larger than the Navier-Stokes value for about $4 \mathrm{fm} / c$. As shown in Fig. 5(a), this leads to the much larger $v_{2}$ suppression of about $10 \%$.

The "critical slowing-down" scenario, which uses a temperature-dependent relaxation time that follows the behavior of $(\zeta / s)(T)$ is shown by the solid and dash-dotted black curves in Fig. 5. In this case the bulk viscous pressure quickly relaxes to its Navier-Stokes value in the interior of the fireball, where the temperature is high and the relaxation time is short; near the edge of the fireball, however, where the temperature is near $T_{c}$ and the relaxation time is long, it remembers its initial value (either zero or the large negative initial Navier-Stokes value) for a long period. With some reflection, one convinces oneself that this implies that, for both zero and Navier-Stokes initializations, the magnitude of the average bulk viscous pressure $\langle\Pi\rangle$ remains below the value observed for the short relaxation time. This is evident in Fig. 5(b) upon comparing the black and green curves. Correspondingly, the viscous $v_{2}$ suppression shown in Fig. 5(a) is, for both initializations, smaller for the criticalslowing-down scenario than for a short constant relaxation time. Comparing the critical-slowing-down scenario with the long constant relaxation time, the viscous $v_{2}$ suppression is significantly smaller for Navier-Stokes initialization $[\mathcal{O}(7 \%)$ vs. $\mathcal{O}(10 \%)]$ and about equally small $[\mathcal{O}(2 \%)]$ for zero initialization.

Because these findings contradict at least our own naive first expectations, we briefly reiterate the main point: taking into account the critical slowing-down of the bulk viscous pressure dynamics near $T_{c}$, where $\zeta / s$ becomes large, leads to weaker bulk viscous suppression effects on the elliptic flow than seen for both short and long constant (i.e., $T$-independent) relaxation times $\tau_{\Pi}$. 


\section{LARGER $\zeta / \mathrm{s}$ AND THE BREAKDOWN OF VISCOUS FLUID DYNAMICS}

As noted in Sec. II, the peak value of $\zeta / s$ in our parametrization shown in Fig. 1 is about 10 times smaller than some other estimates $[65,75]$. When one tries to simply multiply the function shown in Fig. 1 by $C=10$, one finds that (except for special circumstances discussed here) the viscous hydrodynamic code crashes. The reason is that sufficiently large bulk viscosity can lead to fireball regions where the effective total isotropic pressure $p+\Pi$ (thermal + bulk viscous pressure) becomes negative and the medium becomes mechanically unstable and will tend to break up [46-49]. In fact, because certain components of the shear viscous pressure (in particular, its longitudinal component $\pi^{\eta \eta}$ ) are usually also negative, instability can set in even somewhat earlier $[47,48]$. In numerical simulations this manifests itself through the exponential amplification of local numerical errors that will eventually stop the code from running.

We point out that, even before the fluid becomes mechanically unstable, one has left the region of applicability of viscous hydrodynamics. The viscous hydrodynamic formalism is based on a near-equilibrium expansion; its validity assumes that the viscous corrections to the energy-momentum tensor are small compared with the ideal-fluid terms. In other words, if the condition $\left(|\Pi|+\left|\pi^{\mu \nu}\right|\right) /(e+p) \ll 1$ is violated for any component $(\mu \nu)$, the evolution based on Eqs. (2)-(4) can no longer be trusted. Ignoring the shear pressure and setting $e+$ $p=s T \approx 4 p$ for a QGP, the instability threshold $p+\Pi=0$ translates into $|\Pi| /(e+p) \approx \frac{1}{4}$, which is not sufficiently small to trust the continued validity of the equations. The following alternate consideration leads to the same conclusion: if the fluid can be described by quasiparticles, the viscous terms in the energy-momentum tensor correspond to deviations of the local phase-space distribution, $f(x, p)=f_{\text {eq }}+\delta f$, from local equilibrium. Using Grad's 14-moment method, the deviation $\delta f$ is expanded up to quadratic order in momentum $[15,55,56,81,84]$ and (for a fluid with only bulk viscosity and massless particles at midrapidity $y=0$ ) can thus be written in the form

$$
\frac{\delta f}{f_{\mathrm{eq}}}=a \frac{p_{T}^{2}}{T^{2}} \frac{\Pi}{e+p},
$$

where $a$ is a slowly varying function of temperature with magnitude of order unity [84]. When $p+\Pi=0$ such that $\Pi /(e+p)=-\frac{1}{4}$, this means that, for midrapidity particles with typical thermal momenta $p_{T} \simeq 3 T$, the deviation $\delta f / f_{\text {eq }}$ is negative with magnitude 1 or larger, rendering the total distribution function $f$ negative, which is unphysical. Clearly the deviations from local equilibrium are too large and the formalism breaks down.

In this section we explore the range of bulk viscosities that are allowed without leaving the region of validity of second-order (Israel-Stewart) viscous hydrodynamics. As in the preceding section, we study both zero and Navier-Stokes initial conditions and the same three choices for the bulk viscous relaxation time $\tau_{\Pi}$, but we now also include runs where the fluid has an additional shear viscosity $\eta / s=(1 \div 2) /(4 \pi)$, with shear viscous relaxation time $\tau_{\pi}=3 \eta /(s T)$, and we vary

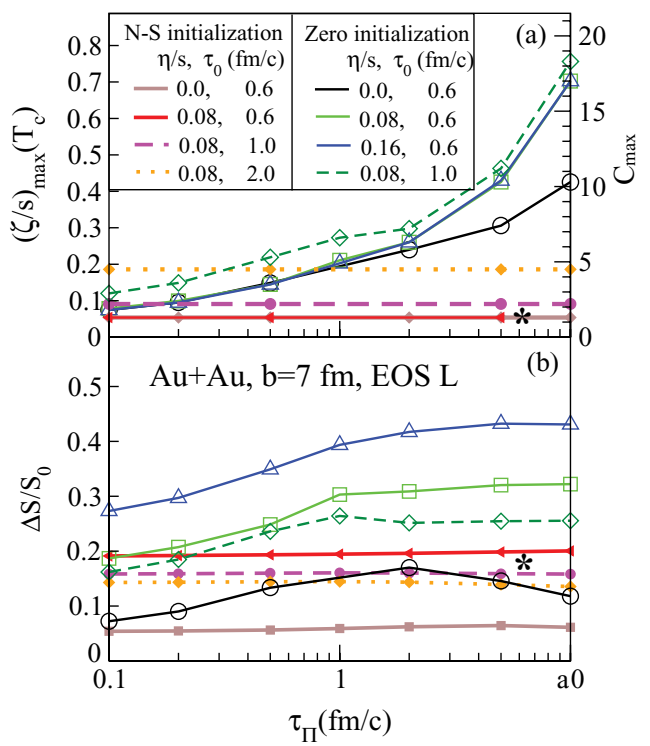

FIG. 6. (Color online) Upper limits for $\zeta / s$ (a) and viscous entropy production (b) as a function of bulk viscous relaxation time $\tau_{\Pi}$, for zero and Navier-Stokes (N-S) initialization. The asterisks indicate the results for the temperature-dependent relaxation time, Eq. (1), with N-S initial conditions, for $\tau_{0}=0.6 \mathrm{fm} / c$. For $\tau_{\Pi}$ given by Eq. (1) and zero initial conditions, there is no upper limit for $\zeta / s$, that is, the fluid remains stable for all values of $C$.

the time $\tau_{0}$ when we start the hydrodynamic evolution. (For later starting times, we downscale the initial peak entropy density $s_{0}$ such that the total initial entropy $\sim s_{0} \tau_{0}$ is held constant.) For the specific bulk viscosity $(\zeta / s)(T)$ we take the functional form shown in Fig. 1 but multiplied by an arbitrary constant $C>1$. For each set of initial conditions, $\tau_{\Pi}$, and $\eta / s$, we determine the largest value of $C_{\max }$ that still allows for stable running of the code, that is, where the effective total isotropic pressure $p+\Pi$ does not violate the stability criterion $p+\Pi>0$ anywhere inside the freeze-out surface.

Figure 6 shows the upper limit $(\zeta / s)_{\max }\left(T_{c}\right)$ (on the left vertical axis) and the corresponding maximal $C$ value, $C_{\max }$ (on the right vertical axis), as a function of the bulk viscous relaxation time $\tau_{\Pi}$. We see that it depends strongly on the initialization.

For Navier-Stokes initial conditions, $(\zeta / s)_{\max }\left(T_{c}\right)$ is insensitive to the relaxation time $\tau_{\Pi}$. In this case the magnitude of the average bulk pressure $\Pi$ decreases more or less monotonically with time [see Fig. 5(b)]. Violations of the positivity condition $p+\Pi>0$ thus always happen at the starting time $\tau_{0}$, at transverse positions where the matter is close to the phase transition. This leads to a $(\zeta / s)_{\max }\left(T_{c}\right)$ that is controlled by the initial conditions and independent of the relaxation time. [This includes the temperature-dependent relaxation time, Eq. (1); see the asterisk in Fig. 6(a).] Correspondingly, $(\zeta / s)_{\max }\left(T_{c}\right)$ does not depend on the value of $\eta / s$ when shear viscosity is included. When one starts the hydrodynamic evolution later, $(\zeta / s)_{\max }\left(T_{c}\right)$ increases with $\tau_{0}$. The dependence on $\tau_{0}$ arises from the strong dependence of the initial bulk pressure, $\Pi=-\zeta \theta=-\zeta \partial \cdot u$, on $\tau_{0}$, through the expansion 
rate $\theta\left(\tau_{0}\right)=1 / \tau_{0}$. This is illustrated by the solid red, dashed magenta, and dotted orange lines in Fig. 6(a): as $\tau_{0}$ is increased from 0.6 to 1 and $2 \mathrm{fm} / c$, the maximal $(\zeta / s)_{\max }\left(T_{c}\right)$ increases from 0.05 to 0.09 and 0.18 .

For zero initialization, $\Pi\left(\tau_{0}\right)=0$, one finds a qualitatively similar dependence of $(\zeta / s)_{\max }\left(T_{c}\right)$ on the starting time $\tau_{0}$ : the curves $C_{\max }\left(\tau_{\Pi}\right)$ move up as $\tau_{0}$ is increased from 0.6 to $1.0 \mathrm{fm} / c$ (solid and dashed green lines). However, in contrast to the Navier-Stokes initialization, the $(\zeta / s)_{\max }\left(T_{c}\right)$ curves now show a strong dependence on relaxation time $\tau_{\Pi}$, rising monotonically with $\tau_{\Pi}$. The reason is that it takes some time for the bulk pressure $\Pi$ to develop large enough magnitudes to violate the positivity condition $p+\Pi>0$; again, this typically happens in regions where the matter is close to the phase transition. For larger relaxation times, $\Pi$ moves away from its zero initial value more slowly, rendering the fluid more stable and resulting in a monotonic increase in $(\zeta / s)_{\max }\left(T_{c}\right)$ with $\tau_{\Pi}$. For $\tau_{\Pi}<1 \mathrm{fm} / c$ we find "universal" $(\zeta / s)_{\max }\left(T_{c}\right)-\tau_{\Pi}$ curves that do not depend on the shear viscosity $\eta / s$ (solid black, green, and blue curves) but move upward as the starting time $\tau_{0}$ is increased. This is because the violation of the positivity condition $p+\Pi>0$ then generally happens at early times, $\tau<3 \mathrm{fm} / c$, when the flow profiles are not yet significantly affected by shear viscous effects. For the two viscous fluid lines with $\eta / s=0.08$ and 0.16 (solid blue and green lines), one sees that they continue to overlap even for $\tau_{\Pi}>1 \mathrm{fm} / c$ after they have broken away from the $\eta / s=0$ line. In the ideal fluid $(\eta / s=0)$ the phase transition generates large velocity gradients near the phase transition that generate locally large expansion rates, causing instability at lower values of $\zeta / s$. Shear viscosity smoothes out these large gradients, as discussed in Ref. [20], allowing the fluid to evolve stably up to larger values of $\zeta / s$. Bulk viscosity $\zeta$ alone has no smoothing influence on sharp structures generated by a phase transition. For a zero initial value of $\Pi$, shear viscosity thus helps crucially in stabilizing the evolution of the viscous fluid against mechanical instabilities caused by strongly negative bulk viscous pressure, especially for large relaxation times $\tau_{\Pi}$.

Very interesting is our finding that, for zero initial conditions, there is no limit on $C_{\max }$ if one accounts for critical slowing-down of the bulk pressure dynamics near $T_{c}$ via Eq. (1). In this case the bulk pressure, starting at zero, never grows sufficiently large to threaten the mechanical stability of the fluid, irrespective of how large the bulk viscosity becomes at $T_{c}$ ! As the peak value $(\zeta / s)\left(T_{c}\right)$ is increased, so is the time it takes $\Pi$ to evolve toward its Navier-Stokes value, and this never happens fast enough to violate the stability condition $p+\Pi>0$.

Figure 6(b) shows the viscous entropy production for the maximally allowed bulk viscosities shown in Fig. 6(a). Not surprisingly, viscous entropy production increases with shear viscosity $\eta / s$ and decreases when hydrodynamics is started later, with correspondingly smaller initial expansion rates [21]. The dependence on $(\zeta / s)_{\max }\left(T_{c}\right)$ is nonmonotonic, however. The reason is that the bulk viscous entropy production rate, $\sim \Pi^{2} /(2 \zeta)$, depends not only on how large $\zeta$ is but also on how close $\Pi$ is to its Navier-Stokes limit, and this in turn depends on $\tau_{\Pi}$.

\section{TOWARD EXTRACTING $\eta$ /s FROM EXPERIMENTAL DATA: UNCERTAINTIES INTRODUCED BY BULK VISCOSITY}

Given the fact that bulk viscosity contributes to the viscous suppression of elliptic flow [see Fig. 5(a)], and assuming that bulk and shear viscous effects cannot be separated by studying other experimental observables, the question naturally arises how much of an irreducible uncertainty this will introduce into the extraction of the specific shear viscosity $\eta / s$ from experimental elliptic flow measurements. More precisely, if the QGP should turn out to be a "most perfect liquid" with "minimal" shear viscosity, $\eta / s=1 / 4 \pi$, with what kind of accuracy can we hope to verify this experimentally if the bulk viscosity is the only quantity beyond our theoretical and experimental control?

To answer this question, we used VISH $2+1$ to compute the differential elliptic flow of directly emitted pions (without resonance decay contributions) for $200-A \mathrm{GeV} \mathrm{Au}+\mathrm{Au}$ collisions at $b=7 \mathrm{fm}$, assuming the fireball medium to have constant specific shear viscosity $\eta / s=1 / 4 \pi$ but allowing the bulk viscosity $\zeta / s$ to vary over the entire range allowed by the mechanical stability criterion $p+\Pi>0$. In doing so we assumed a fixed shape of the temperature dependence of $\zeta / s$ as shown in Fig. 1 but let its normalization vary between $C=1$ and $C_{\max }\left(\tau_{\Pi}\right)$, where the latter is the largest value within the range of applicability of Israel-Stewart viscous fluid dynamics, shown in Fig. 6(a). We allowed for two fixed values of 0.5 and $5 \mathrm{fm} / c$ for the bulk viscous relaxation time $\tau_{\Pi}$ as well as for "critical slowing-down" according to Eq. (1), and we studied both zero and Navier-Stokes initial values for the viscous pressure components. All calculations assume $\tau_{0}=0.6 \mathrm{fm} / c$ as the starting time. The results are presented in Fig. 7 and Table I.

Generically one observes that, even for minimal shear viscosity near the KSS bound, the shear viscous contribution to the elliptic flow suppression far exceeds the bulk viscous contribution. This is good news, as it means that the uncertainty introduced into the extraction of $\eta / s$ by theoretically poorly controlled bulk viscous effects remains limited and is, in fact, quite small, especially if the real fireballs created in heavy-ion collisions do not completely saturate the KSS bound. On a more quantitative level, one finds that, for pions with typical transverse momentum $p_{T}=0.5 \mathrm{GeV} / c$, the elliptic flow is suppressed by just over $16 \%$ below the ideal-fluid value if the expanding matter has only shear, and no bulk, viscosity and that this suppression increases to values between $17 \%$ and $25 \%$ if bulk viscosity is added. The largest bulk viscous suppression is found for fixed relaxation times $\tau_{\Pi}$ and zero initialization if the bulk viscosity is increased all the way up to its upper allowed limit. In these cases the additional suppression can be as large as 50\% of the suppression found for the fluid with only (minimal) shear viscosity. If one takes into account that the evolution of the bulk viscous pressure slows down near $T_{c}$, where $\zeta / s$ is largest, the additional bulk viscous suppression never exceeds $20 \%$ of the shear viscous elliptic flow suppression, with $10 \%-15 \%$ being a typical range (aqua and green curves in Fig. 7). 
TABLE I. Pion elliptic flow at $p_{T}=0.5 \mathrm{GeV} / c$ for $b=7 \mathrm{fm}, 200-A \mathrm{GeV} \mathrm{Au}+\mathrm{Au}$ collisions from ideal and viscous hydrodynamics, with different choices of initial conditions, bulk viscous relaxation times $\tau_{\Pi}$, and bulk viscosities (parametrized by $C$ ). The last of the three columns under each initialization heading gives the viscous suppression of $v_{2}$ at $p_{T}=0.5 \mathrm{GeV} / c$ in terms of the percentage deviation from the ideal fluid baseline $(=100 \%)$.

\begin{tabular}{|c|c|c|c|c|c|c|c|}
\hline \multirow[t]{2}{*}{$\eta / s$} & \multirow[t]{2}{*}{$\tau_{\Pi}(\mathrm{fm} / c)$} & \multicolumn{3}{|c|}{ Zero initialization } & \multicolumn{3}{|c|}{ Navier-Stokes initialization } \\
\hline & & C & $v_{2}(0.5 \mathrm{GeV} / c)(\%)$ & $\frac{v_{2}}{v_{2, \text { ideal }}}(\%)$ & $C$ & $v_{2}(0.5 \mathrm{GeV} / c)(\%)$ & $\frac{v_{2}}{v_{2, \text { ideal }}}(\%)$ \\
\hline 0 & - & 0 & 5.755 & 100 & 0 & 5.755 & 100 \\
\hline 0.08 & - & 0 & 4.821 & 83.8 & 0 & 4.811 & 83.6 \\
\hline 0.08 & 0.5 & 1 & 4.668 & 81.1 & 1 & 4.627 & 80.4 \\
\hline 0.08 & 0.5 & 3.5 & 4.356 & 75.7 & 1.3 & 4.576 & 79.5 \\
\hline 0.08 & 5.0 & 1 & 4.770 & 82.9 & 1 & 4.601 & 79.9 \\
\hline 0.08 & 5.0 & 10.3 & 4.323 & 75.1 & 1.3 & 4.534 & 78.8 \\
\hline 0.08 & Eq. (1) & 1 & 4.743 & 82.5 & 1 & 4.660 & 81.0 \\
\hline 0.08 & Eq. (1) & 100 & 4.656 & 80.9 & 1.3 & 4.615 & 80.2 \\
\hline
\end{tabular}

An important caveat, however, is that for Navier-Stokes initial conditions the allowed maximal bulk viscosities are small, much below recent lattice QCD estimates [75]. If larger values are realized by Nature, they invalidate the use of viscous hydrodynamics, at least at early times [86-89]. The problems in this case arise from the large bulk viscosity in a thin layer near the transverse edge of the fireball, where the matter is close to $T_{c}$. It is only in this region that the viscous hydrodynamic description breaks down. Because the problematic factor, the scalar expansion rate $\theta$, initially

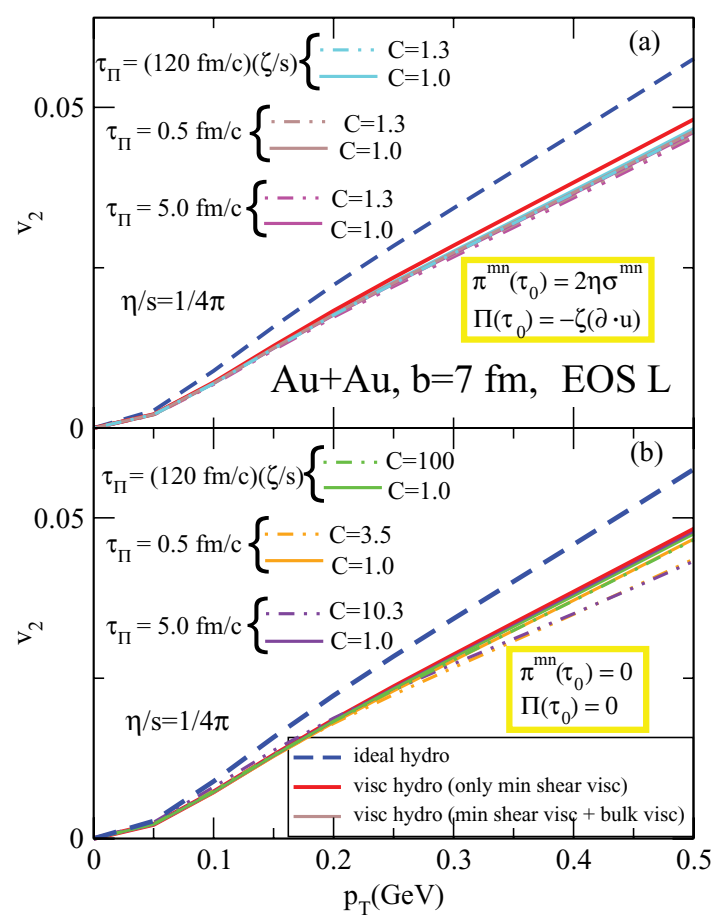

FIG. 7. (Color online) $v_{2}\left(p_{T}\right)$ for directly emitted pions from ideal and viscous hydrodynamics with Navier-Stokes (a) or zero (b) initial conditions for viscous pressures. Results for minimal shear viscosity $\eta / s=1 / 4 \pi$ and bulk viscosities ranging from "minimal" $(C=1)$ to the maximal values from Fig. 6(a) that still allow for stable viscous evolution, for three choices of bulk viscous relaxation time $\tau_{\Pi}$. decreases very rapidly, these initially unstable fluid regions move quickly back to mechanical stability. As the momentum anisotropy does not develop instantaneously, we find it hard to believe that the existence of this unstable external layer has much influence on the evolution and final value of the elliptic flow, and one should get very similar results from simulations in which the initial bulk viscous pressure $\Pi$ is restricted by hand to values below the threshold for violating the positivity condition $p+\Pi>0$. If this is indeed the case, the results presented in this section show that bulk viscosity, even if theoretically not well controlled, will not introduce large uncertainties into the extraction of $\eta / s$ from elliptic flow data.

\section{CONCLUDING REMARKS}

The present study has shown that bulk viscosity-as long as it is small enough that, in expanding heavy-ion collision fireballs, the negative bulk viscous pressure does not become larger than the thermodynamic pressure-affects the elliptic flow of the final hadrons much more weakly than does shear viscosity. So, as long as the expanding fireball can be described by viscous fluid dynamics, it is possible to extract its shear viscosity (even if it is as small as $\left.\frac{\eta}{s}\right|_{\text {KSS }}=\frac{1}{4 \pi}$ ) with good accuracy from a comparison of viscous hydrodynamic simulations with experimental elliptic flow data. Accounting for the critical slowing-down of viscous bulk pressure dynamics near $T_{c}$, we have shown that any contamination from bulk viscosity $\zeta / s$ is $<20 \%$ (for much of the parameter space it is even $<10 \%$ ) and that its relative importance decreases further if $\eta / s$ is larger than the KSS bound.

However, we also saw that the stability condition $p+\Pi>$ 0 is very restrictive and easily violated if the peak value of $\zeta / s$ near $T_{c}$ reaches values close to those estimated from lattice QCD [75] and from some strong coupling approaches [65], and if the bulk viscous pressure $\Pi$ approaches its Navier-Stokes limit $\Pi=-\zeta \partial \cdot u$. When this occurs (typically at early times, when the scalar expansion rate is largest, in a thin layer around $T_{c}$ close to the transverse edge of the fireball), the viscous fluid dynamical description breaks down. Our analysis shows that the phenomenon of "critical slowing-down" can play a 
crucial role in preventing this from happening. Kinetic theory for weakly coupled systems [55,90] and a recent analysis of strongly coupled systems by Buchel [65] suggest that the same microscopic physics (namely, growing correlation lengths owing to critical fluctuations) that generate a peak of $\zeta / s$ at $T_{c}$ also causes the relaxation time $\tau_{\Pi}$ for the bulk viscous pressure to grow and possibly diverge at $T_{c}$, even while $\zeta / s$ itself remains finite. When using the model Eq. (1) for a temperature-dependent $\tau_{\Pi}$ inspired by these ideas, we saw that, unless $\Pi$ is initialized at its Navier-Stokes limit, it never reaches it during the short time span of a heavy-ion collision in those fireball regions where $\zeta / s$ peaks and $\Pi$ could thus become very large. This reduces the problem of applicability of viscous hydrodynamics at early times to a question of initial conditions for $\Pi$, especially in that thin transverse layer where (after local equilibrium is reached) the temperature happens to be close to $T_{c}$.

Determining these initial conditions (as opposed to guessing them, as we have done here) requires a theoretical description of the early pre-equilibrium evolution and Landau matching of the corresponding energy-momentum tensor to its viscous fluid dynamic form, Eq. (2) (in the spirit of Ref. [89] but generalized from $0+1$ to $2+1$ dimensions). At this point we lack the tools for doing this. Let us, however, make a few comments in anticipation of completion of that task. Consider a small fireball region that is just reaching local thermal equilibrium at a temperature close to $T_{c}$ and undergoing self-similar boost-invariant longitudinal expansion, while transverse expansion is negligible. Let us also assume that at this point in time the bulk viscous pressure in the region is large and negative, leading to negative effective total isotropic pressure and causing the fluid to be mechanically unstable. What will happen? The fluid will begin to rupture, forming little voids, and if the region were to remain in a state of negative total pressure, it would eventually fragment. However, as the considered region is undergoing rapid expansion and cooling, it will quickly exit from its state of mechanical instability. Furthermore, during the short period of instability the hydrodynamic growth of voids will be hampered by the large value of the relaxation time $\tau_{\Pi}$. By the time the considered region becomes mechanically stable again, we expect it to be riddled with small holes but otherwise intact. The small voids formed during the period of instability will recollapse by cavitation, and the region will quickly re-equilibrate owing to the now much shorter relaxation time below $T_{c}$. No wholesale breakup of the fluid will occur, owing to lack of time. Similar arguments hold later, when the bulk of the matter in the center of the fireball passes through $T_{c}$, only, in this case, the viscous bulk pressure may never grow large enough to generate mechanical instability, owing to critical slowing-down.

In summary, unlike the authors of Ref. [48], we do not expect any dramatic macroscopic phenomena triggered by the transient mechanical instability arising from possibly large, but short-lived negative bulk pressures in fireball regions passing through the hadronization phase transition. For this reason we believe that a modified viscous hydrodynamic treatment, where one limits by hand the growth of the viscous bulk pressure so that it always remains below the instability threshold [91], will not lead to impermissible distortions of the real (nonequilibrium) dynamics in the (small) space-time regions whose description lies outside the hydrodynamic domain. This is important for future viscous hydrodynamic studies of heavy-ion collisions with fluctuating and granular initial conditions [92] that are more realistic than the smooth initial profiles presently used.

\section{ACKNOWLEDGMENTS}

We gratefully acknowledge informative discussions with K. Dusling, P. Petreczky, and J. Randrup and thank T. Hirano and G. Moore for constructive comments on the manuscript. U.H. is indebted to K. Rajagopal for a very fruitful exchange of ideas that clarified much of the argument presented in the last section of the paper. This work was supported by the US Department of Energy under Contract No. DE-FG0201ER41190.
[1] P. F. Kolb and U. Heinz, in Quark-Gluon Plasma 3, edited by R. C. Hwa and X.-N. Wang (World Scientific, Singapore, 2004), p. 634.

[2] T. Hirano, U. Heinz, D. Kharzeev, R. Lacey, and Y. Nara, Phys. Lett. B636, 299 (2006).

[3] D. Teaney, J. Lauret, and E. V. Shuryak, arXiv:nucl-th/0110037; Nucl. Phys. A698, 479 (2002).

[4] U. Heinz and P. F. Kolb, Nucl. Phys. A702, 269 (2002).

[5] M. Gyulassy, in Structure and Dynamics of Elementary Matter, edited by W. Greiner et al., NATO Science Series II: Mathematics, Physics and Chemistry, Vol. 166 (Kluwer Academic, Dordrecht, 2004), pp. 159-182.

[6] M. Gyulassy and L. McLerran, Nucl. Phys. A750, 30 (2005).

[7] E. V. Shuryak, Nucl. Phys. A750, 64 (2005).

[8] P. Danielewicz and M. Gyulassy, Phys. Rev. D 31, 53 (1985).

[9] G. Policastro, D. T. Son, and A. O. Starinets, Phys. Rev. Lett. 87, 081601 (2001).
[10] P. K. Kovtun, D. T. Son, and A. O. Starinets, Phys. Rev. Lett. 94, 111601 (2005).

[11] L. P. Csernai, J. I. Kapusta, and L. D. McLerran, Phys. Rev. Lett. 97, 152303 (2006).

[12] T. Schäfer, Phys. Rev. A 76, 063618 (2007).

[13] L. Luo, B. Clancy, J. Joseph, J. Kinast, and J. E. Thomas, Phys. Rev. Lett. 98, 080402 (2007); B. Clancy, L. Luo, and J. E. Thomas, ibid. 99, 140401 (2007); A. Turlapov et al., J. Low Temp. Phys. 150, 567 (2008); J. E. Thomas, Nucl. Phys. A830, 665c (2009).

[14] U. Heinz and S. M. H. Wong, Phys. Rev. C 66, 014907 (2002).

[15] D. Teaney, Phys. Rev. C 68, 034913 (2003).

[16] U. Heinz, H. Song, and A. K. Chaudhuri, Phys. Rev. C 73, 034904 (2006).

[17] P. Romatschke and U. Romatschke, Phys. Rev. Lett. 99, 172301 (2007).

[18] H. Song and U. Heinz, Phys. Lett. B658, 279 (2008).

[19] K. Dusling and D. Teaney, Phys. Rev. C 77, 034905 (2008). 
[20] H. Song and U. Heinz, Phys. Rev. C 77, 064901 (2008).

[21] H. Song and U. Heinz, Phys. Rev. C 78, 024902 (2008).

[22] M. Luzum and P. Romatschke, Phys. Rev. C 78, 034915 (2008); 79, 039903(E) (2009).

[23] D. Molnar and P. Huovinen, J. Phys. G 35, 104125 (2008).

[24] E. Molnar, H. Niemi and D. H. Rischke, arXiv:0907.2583 [nucl-th].

[25] D. Teaney, Prog. Part. Nucl. Phys. 62, 451 (2009).

[26] U. Heinz, arXiv:0901.4355 [nucl-th].

[27] P. Romatschke, arXiv:0902.3663 [hep-ph].

[28] D. A. Teaney, arXiv:0905.2433 [nucl-th].

[29] R. A. Lacey and A. Taranenko, PoS CFRNC2006, 021 (2006).

[30] R. A. Lacey et al., Phys. Rev. Lett. 98, 092301 (2007).

[31] H. J. Drescher, A. Dumitru, C. Gombeaud, and J. Y. Ollitrault, Phys. Rev. C 76, 024905 (2007).

[32] Z. Xu, C. Greiner, and H. Stocker, Phys. Rev. Lett. 101, 082302 (2008).

[33] Z. Xu and C. Greiner, Phys. Rev. C 79, 014904 (2009).

[34] H. Song and U. Heinz, J. Phys. G 36, 064033 (2009).

[35] A. Adil, H.-J. Drescher, A. Dumitru, A. Hayashigaki, and Y. Nara, Phys. Rev. C 74, 044905 (2006).

[36] H.-J. Drescher and Y. Nara, Phys. Rev. C 75, 034905 (2007).

[37] R. A. Lacey, A. Taranenko, and R. Wei, in Proceedings, 25th Winter Workshop on Nuclear Dynamics, edited by W. Bauer, R. Bellwied and J. W. Harris (EP Systema, Budapest, 2009), p. 73 .

[38] U. Heinz, J. S. Moreland, and H. Song, Phys. Rev. C 80, 061901(R) (2009).

[39] P. F. Kolb and R. Rapp, Phys. Rev. C 67, 044903 (2003).

[40] D. Teaney, arXiv:nucl-th/0204023.

[41] T. Hirano and K. Tsuda, Phys. Rev. C 66, 054905 (2002).

[42] T. Hirano and M. Gyulassy, Nucl. Phys. A769, 71 (2006).

[43] P. Huovinen, Eur. Phys. J. A 37, 121 (2008).

[44] H. Song and U. Heinz, Nucl. Phys. A830, 467c (2009).

[45] G. S. Denicol, T. Kodama, T. Koide, and Ph. Mota, Phys. Rev. C 80, 064901 (2009); Nucl. Phys. A830, 729c (2009).

[46] G. Torrieri and I. Mishustin, Phys. Rev. C 78, 021901(R) (2008).

[47] R. J. Fries, B. Müller, and A. Schäfer, Phys. Rev. C 78, 034913 (2008).

[48] K. Rajagopal and N. Tripuraneni, arXiv:0908.1785 [hep-ph].

[49] G. Torrieri, B. Tomášik, and I. Mishustin, Phys. Rev. C 77, 034903 (2008).

[50] J. I. Kapusta, arXiv:0809.3746 [nucl-th].

[51] J. Frenkel, Kinetic Theory of Liquids (Dover, Mineola, NY, 1955); O. Hirshfelder, C. Curtis, and R. Bird, Molecular Theory of Gases and Liquids (Wiley Interscience, New York, 1964).

[52] H. B. Meyer, Phys. Rev. D 76, 101701(R) (2007).

[53] P. Arnold, G. D. Moore, and L. G. Yaffe, J. High Energy Phys. 05 (2003) 051.

[54] N. Demir and S. A. Bass, Phys. Rev. Lett. 102, 172302 (2009).

[55] W. Israel, Ann. Phys. (NY) 100, 310 (1976); W. Israel and J. M. Stewart, ibid. 118, 341 (1979).

[56] R. Baier, P. Romatschke, and U. A. Wiedemann, Phys. Rev. C 73, 064903 (2006).

[57] M. A. York and G. D. Moore, Phys. Rev. D 79, 054011 (2009).
[58] H. B. Meyer, Nucl. Phys. A830, 641c (2009).

[59] R. Baier, P. Romatschke, D. T. Son, A. O. Starinets, and M. A. Stephanov, J. High Energy Phys. 04 (2008) 100.

[60] S. Bhattacharyya, V. E. Hubeny, S. Minwalla, and M. Rangamani, J. High Energy Phys. 02 (2008) 045.

[61] M. Natsuume and T. Okamura, Phys. Rev. D 77, 066014 (2008); 78, 089902(E) (2008).

[62] A. Onuki, Phys. Rev. E 55, 403 (1997).

[63] K. Paech and S. Pratt, Phys. Rev. C 74, 014901 (2006).

[64] G. D. Moore and O. Saremi, J. High Energy Phys. 09 (2008) 015.

[65] A. Buchel, Phys. Lett. B681, 200 (2009).

[66] S. Gavin, Nucl. Phys. A435, 826 (1985).

[67] S. Weinberg, Astrophys. J. 168, 175 (1971).

[68] P. Arnold, C. Dogan, and G. D. Moore, Phys. Rev. D 74, 085021 (2006).

[69] A. Buchel, Phys. Lett. B663, 286 (2008).

[70] M. Prakash, M. Prakash, R. Venugopalan, and G. Welke, Phys. Rep. 227, 321 (1993).

[71] D. Davesne, Phys. Rev. C 53, 3069 (1996).

[72] J. W. Chen and J. Wang, Phys. Rev. C 79, 044913 (2009).

[73] D. Kharzeev and K. Tuchin, J. High Energy Phys. 09 (2008) 093; F. Karsch, D. Kharzeev, and K. Tuchin, Phys. Lett. B663, 217 (2008).

[74] S. D. Katz, Nucl. Phys. A774, 159 (2006).

[75] H. B. Meyer, Phys. Rev. Lett. 100, 162001 (2008).

[76] U. Gursoy, E. Kiritsis, G. Michalogiorgakis, and F. Nitti, J. High Energy Phys. 12 (2009) 056.

[77] S. S. Gubser, A. Nellore, S. S. Pufu, and F. D. Rocha, Phys. Rev. Lett. 101, 131601 (2008); S. S. Gubser, S. S. Pufu, and F. D. Rocha, J. High Energy Phys. 08 (2008) 085.

[78] A. Muronga, Phys. Rev. Lett. 88, 062302 (2002); 89, 159901(E) (2002); Phys. Rev. C 69, 034903 (2004).

[79] A. Muronga and D. H. Rischke, arXiv:nucl-th/0407114.

[80] A. Muronga, Phys. Rev. C 76, 014909 (2007).

[81] B. Betz, D. Henkel, and D. H. Rischke, J. Phys. G 36, 064029 (2009).

[82] https://wiki.bnl.gov/TECHQM/index.php/Code_verification_for -viscous_hydrodynamics (in preparation).

[83] P. F. Kolb, J. Sollfrank, and U. Heinz, Phys. Lett. B459, 667 (1999); Phys. Rev. C 62, 054909 (2000).

[84] A. Monnai and T. Hirano, Phys. Rev. C 80, 054906 (2009).

[85] P. Romatschke, Eur. Phys. J. C 52, 203 (2007).

[86] A. Dumitru, E. Molnar, and Y. Nara, Phys. Rev. C 76, 024910 (2007).

[87] P. Huovinen and D. Molnar, Phys. Rev. C 79, 014906 (2009).

[88] M. Martinez and M. Strickland, Phys. Rev. C 79, 044903 (2009)

[89] M. Martinez and M. Strickland, arXiv:0909.0264 [hep-ph].

[90] S. R. De Groot, W. A. Van Leeuwen, and C. G. Van Weert, Relativistic Kinetic Theory. Principles and Applications (North-Holland, Amsterdam, 1980).

[91] S. Pratt, Phys. Rev. C 77, 024910 (2008).

[92] J. Takahashi, B. M. Tavares, W. L. Qian, R. Andrade, F. Grassi, Y. Hama, T. Kodama, and N. Xu, Phys. Rev. Lett. 103, 242301 (2009). 